\title{
Studies on Relationships between Actinophage and Variation in Streptomyces
}

\section{Morphology of Phage Production and Lysis in a Streptomycete Carrying a Temperate Phage}

\author{
Elwood B. SHIRLING ${ }^{1,2}$ \\ Department of Botany, University of Michigan, Ann Arbor, Michigan
}

Accepted January 27, 1956

The relationship of phage production to lysis and variation in a streptomycete carrying temperate phage was investigated. Macroscopically, the original isolate, $\mathrm{S}-77$, produced cartilaginous mycelial growth characteristic of Streptomyces. Microscopic examination, however, showed that discrete portions of hyphae were lysed within 48 hours of growth. These lysed portions were widely spaced with the ghost hyphal walls intact so that the mycelial structure and sporogenous habit of the streptomycete were maintained. Variants occurred which ranged from essentially normal-appearing cultures, through lightly sporulated and friable intermediates, to completely asporogenous and mucid cultures.

Filtration and electron microscope studies established the presence of phage and showed ghost areas in hyphae as sites of phage release. Phage was found in filtrates of all S-77 variants including the normal-appearing parent type. Incomplete phage particles consisting of collapsed head membranes and of tailless particles were also common. Soft colony types with numerous ghost areas released large amounts of phage. Perfect phage was diffeult to find in macroscopically normal cultures where Iysed areas were widely spaced. Both normal S-77 and soft variants are either lysogenic or "carriers," since host and phage coexist in an equilibrial state through repeated transfer.

It is suggested that the striking variation in morphological and cultural characteristics observed in $\mathbf{5 - 7 7}$ results from shifts in the host-phage equilibrium.

Actinophage in Streptomyces griseus fermentations was shown by Carvajal (1953) to result in a striking variety of colony types. Colonies

1 This paper is based in part on the author's Doctoral thesis deposited in the University of Michigan library.

${ }^{2}$ Present address, Department of Botany, Ohio Wesleyan University, Delaware, Ohio. 
arising as survivors after phage action displayed wide variation in morphological, cultural, and biochemical characteristics. The frequent occurrence of comparable variation accompanying lytic phenomena among Streptomyces recently isolated from the soil has suggested that phage may be an important contributor to the distressing variability within this genus.

In order to examine more critically the role of phage in seeming instability of streptomycetes, a single soil isolate showing a wide range of variation has been selected for intensive study. This paper deals with phage release and morphology of lysis in variants of this isolate in which variation is believed to be related to shifts in host-phage equilibrium.

\section{MATERIALS AND METHODS}

Cullures. Streplomyces S-77 used in this work is a soil isolate from the collection of Dr. K. L. Jones, University of Michigan. This isolate is known to produce a wide range of variants some of which are soft, collapsed, and asporogenous. S-77L and $\mathrm{S}-77 \mathrm{~F}$ are representative of collapsed variants and served as sources of phage.

Culture methods. Difco nutrient-agar plus 2 per cent glycerol was used in studies requiring an agar base. Shaken broth cultures were grown in $125 \mathrm{ml}$ flasks containing $50 \mathrm{ml}$ of Difco nutrient-broth plus 2 per cent glycerol. All media were adjusted to $\mathrm{pH} 7.6$ before autoclaving. Shaken cultures were grown on a reciprocating shaker with seven-cmtravel, 90 strokes per minute. Incubation was at room temperature.

Electron microscope studies. Observations were made in an RCA model EMB electron microscope. In shadow-cast preparations, uranium was the shadowing element. Mycelium scraped from an agar surface was fragmented artificially by grinding lightly in 0.4 per cent saline between sterile slides, washed into a centrifuge tube with $5 \mathrm{ml}$ of the saline, and the volume was brought up to $20 \mathrm{ml}$ by slow drop-by-drop addition of sterile distilled water.

Cultures of S-77 grown on the shaker for 30 hours also consisted of mycelial clumps unsuitable for electron microscopy. It was found that these could be artificially fragmented by ejecting repeatedly through a pipette with a fine tip opening. In shaken cultures of S-77L and $\mathrm{S}-77 \mathrm{~F}$ extensive lytic breakdown of the mycelium made artificial fragmentation unnecessary; no large mycelial clumps remained in 30-hour cultures. In each instance the suspension of mycelial fragments was washed three times in sterile distilled water by low speed centrifugation. Following 
the last wash the volume of water replaced was adjusted to give a suitable concentration of fragments in droplets placed on the collodion membrane.

Phage. Some electron micrographs of the washed mycelial preparations described above revealed phage particles. Partially purified and concentrated phage was obtained by the following method. Lysed content of a 4-day shaken culture flask of the streptomycete was centrifuged to remove some mycelial material and debris and the supernatant further purified by filtration through a Selas 03 porosity porcelain filter. The phage in this supernatant was sedimented in an ultracentrifuge at $20,000 \mathrm{~g}$ for 30 minutes. (A small bright orange fluorescent pellet was formed from $\mathrm{S}-\mathbf{7 7} \mathrm{F}$ lysates, but $\mathrm{S}-77$ did not give a visible pellet.) Following centrifugation the supernatant was resuspended in $0.5 \mathrm{ml}$ of twice distilled water. Droplet patterns on collodion-filmed grids for size measurement and particle counts were prepared by a modification of the spray techniques of Backus and Williams (1950); Luria et al. (1951).

\section{RESULTS}

Variants. Isolate S-77 as obtained from the soil was macroscopically a normal streptomycete (Fig. 1). It produced a true-branching vegetative mycelium which formed cartilaginous growth that was adherent to the substrate. A typical dense aerial mycelium consisting of whorled tufts of erect, branched, nonspiraled filaments and gray cylindrical spores developed after 2 to 5 days on organic media. Individual hyphae of the young substrate mycelium had occasional segments devoid of protoplasm that appeared as clear gaps when stained with Loeffler's methylene blue or when examined in a living state with the phase contrast microscope. Electron micrographs show these as ghost hyphal walls connecting normal sections of the mycelium (Fig. 6). They appear to be sites of phage release (Fig. 9). True fragmentation of the young mycelium as found in Nocardia did not occur. The substrate mycelium produced soluble pigment which was at first golden-yellow but later became brown. Shaken cultures in glycerol-nutrient-broth produced irregular clumps and floccules of submerged growth, some as large as a pea, with no turbidity of the broth. A brown melanistic pigment was formed.

Variant S-77L showing rather rapid and extensive lytic disruption of the mycelium, appeared "spontaneously" as a single subculture in one of many stock glycerol-nutrient-agar slants of S-77. During the first 24 to 48 hours of growth on agar media the two strains were indistinguishable. Lysis then altered the pattern of development in S-77L. Aerial 

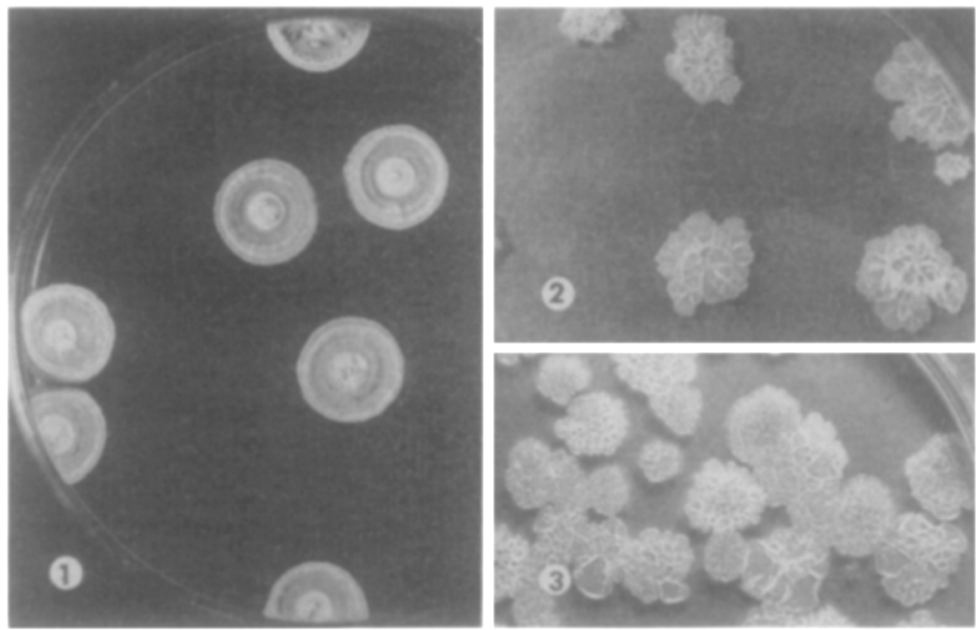

FIg. 1. Streptomyces isolate S-77. Normal colonies at 1 week. Note symmetrical growth, dense acrial eover, grayed chalky appcarance indicating conidia forma tion. Such colonies are coriaceous and adherent to the agar.

FIa. 2. Variant S-77L at 1 week. Colonies lack aerial growth and spores, are emulsive to butyrous, and do not adhere to the agar.

FIg. 3. Variant S-77F. Another collapsed variant obtained by mass subculture of surviving growth when S-77 was grown on agar in the presence of S-77L phage.

mycelium and spores were not formed. Macroscopically the growth became highly rugose and soft, resembling cultures of Nocardia (Fig. 2). Pigment formation was the same as in the original strain. The softened vegetative growth, which no longer adhered to the substrate, consisted of fragments of various sizes surviving extensive lysis of portions of the mycelium. The fragments were usually viable. Shaken cultures became densely turbid in 24 to 48 hours primarily as a result of suspension of short living fragments of the mycelium, but in part from lytic debris. This variant type was found among the progeny in a series of single spore or fragment isolations using only normal S-77 type colonies as a source of inoculum. In plating experiments, repeated selection of inoculum from sporulated S-77 type colonies has never given less than 1 per cent lytic, asporogenous (S-77L type) colonies.

The origin of variant S-77F (Fig. 3) is discussed in the following section. Like S-77L it undergoes rather rapid and extensive lysis resulting in a soft-rugose macroscopic growth and failure to develop aerial mycelium. In shaken broth culture, this variant is somewhat more lytic 
TABLE 1

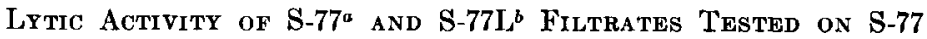
and Two Unrelated Streptomycetes

\begin{tabular}{lccc}
\hline \multicolumn{1}{c}{ Filtrate source } & Quantity $^{c}$ & Test strain & Lytic effect $^{\text {d }}$ \\
\hline none & none & $\mathrm{S}-77$ & - \\
S-77 & 0.1 & $\mathrm{~S}-77$ & - \\
S-77 & 1.0 & $\mathrm{~S}-77$ & - \\
S-77L & 0.1 & $\mathrm{~S}-77$ & + \\
S-77L & 1.0 & $\mathrm{~S}-77$ & ++ \\
S-77L (heated & 1.0 & $\mathrm{~S}-77$ & - \\
90C/10 min.) & & & (growth enhanced) \\
S-77L & 1.0 & 3-d & - \\
S-77L & 1.0 & Streptomyces & - \\
& & griseus & \\
\hline
\end{tabular}

"So-called "normal" or relatively nonlytic strain.

"So-called "lytic" strain.

${ }^{c}$ Milliliters of filtrate per $5 \mathrm{ml}$ of medium.

$d$ Lytic effect as indicated by clearing of growth. Three plus $(+++)$ indicates almost complete clearing of growth except for a few scattered resistant clumps at 48 hours. One plus (+) indicates some clearing, but numerous nonlysed clumps. Addition of heat denatured filtrate actually stimulated a more luxuriant aerial growth of $\mathbf{S - 7 7}$. Minus (-) indicates no macroscopic evidence of lysis.

than S-77L. Turbid cultures with no submerged mycelial clumps are formed. It fails to develop the brown pigment shown by S-77 and S-77L on organic media.

Lytic activity of filtrates. Cell-free filtrates containing phage from S-77 and $\mathrm{S}-77 \mathrm{~T}_{2}$ were self-tested against the homologous strain and cross tested for effect on the opposing strain. Addition of phage-containing filtrates brought no increases in lysis when the soft $\mathrm{S}-77 \mathrm{~L}$ was used as the test strain. When the parent S-77 was used as the test culture, activity was as shown in Table 1. Although typical phage plaques were not produced in this type of experiment the activity of filtrates could be observed qualitatively on heavily seeded plates or slants. All slants showed a good initiation of tough, adherent mycelial growth at 15 hours with no difference between controls and slants containing active filtrate. After 24 hours all controls were covered with characteristic tough and adherent growth; S-77 slants containing $0.1 \mathrm{ml}$ of $\mathrm{S}-77 \mathrm{~L}$ filtrate showed a partial clearing of growth between numerous opaque surviving clumps; and slants containing $1.0 \mathrm{ml}$ of filtrate had only a mottled, soft, and nearly transparent film of lysed material over much of the surface, with a few 
widely scattered resistant clumps of opaque growth. Lysis was accompanied by an increase in melanistic pigment which diffused into the medium.

Microscopic observation $(440 \times)$ of blocks of agar cut from Petri plates heavily seeded with S-77 and treated with filtrates as described for the slants, showed that the clearing of growth after 24 hours was due to progressive lysis of segments of young radiating mycelium. In 48 hours, each surviving center of growth on the filtrate-treated cultures was surrounded by mycelial fragments of varying length interrupted by lysed gaps of $10 \mu$ to $30 \mu$. Subsequent observation showed additional destruction followed, however, by limited sporulating regrowth from surviving fragments. When such resistant growth was used as inoculum for subculture, the resulting growth was slow to develop, nearly asporogenous, mottled with soft pasty areas. In the second and third subcultures softened areas became progressively more prominent and aerial hyphae failed to appear. After four transfers a relatively stable variant which we have designaled S-77F (Fig. 3) was derived having morphological properties closely resembling the filtrate source, S-77L. Filtrates from S-77F were tested with results similar to those described for S-77L.

As might be anticipated from these experiments on agar, the action of filtrate from either of the lytic variants, S-77L or $\mathrm{S}-77 \mathrm{~F}$ on shaken broth-culture of S-77 increased lysis of the test culture, but did not result in complete clearing of the culture. Because of the mycelial nature of streptomycetes, shaken broth-cultures are normally free of turbidity; characteristic growth consists of small spherical or granular colonies of macroscopic dimensions submerged in clear broth. The effect of filtrate from S-77L and S-77F on shaken broth-culture of S-77, however, was to produce extreme opaque turbidity of the culture in place of the usual granular or flocculent submerged growth.

Microscopic examination showed the turbidity to be of the same type observed in broth cultures of the lytic variants, and to be the direct consequence of mycelial breakdown following irregular and scattered lysis of segments within the hyphae. Most of the turbidity was due to short surviving fragments, branched and unbranched, and often distended and pleomorphic, although lytic debris also contributed to the clouding. Part of this material was viable and gave rise to a variety of colony types.

Electron microscope observations of progression of lysis in variants. The essentially uninterrupted nature of the mycelium of both S-77 and S-77F 

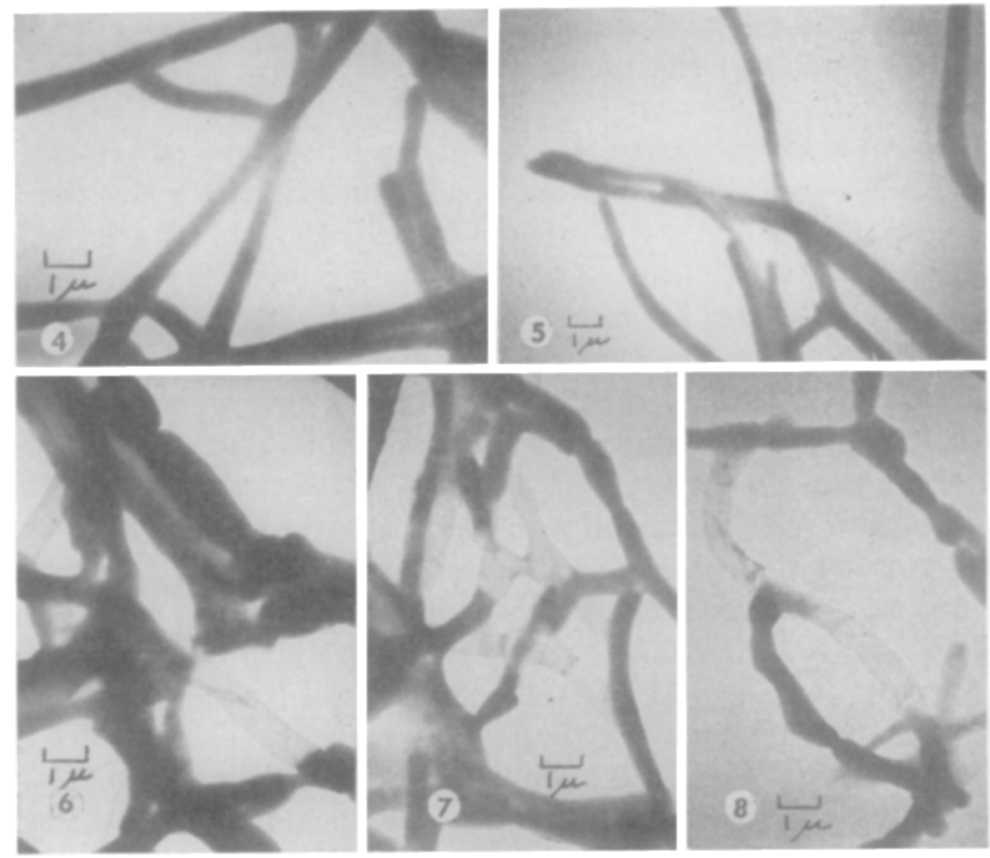

Fig. 4. Normal, unlysed mycelium of S-77 at 24 hours. Electron micrograph, not shadowed, $4500 \times$.

Fig. 5. Mycelium of S-77F showing no lysis at 24 hours. Electron micrograph, not shadowed, $3750 \times$.

Fig. 6. S-77, 48 hours. Occasional ghost segments in normal mycelium at probable sites of phage release. Ghost segments are widely separated in S-77. Electron micrograph, not shadowed, $4500 \times$.

Figs. 7 and 8 . S-77F, 2-3 days. Much of the mycelium is reduced to ghost walls. Surviving hyphae show tendency toward segmentation, are easily fragmented, and are generally viable. Flectron micrographs, not shadowed, $4500 \times$.

during the first 24 hours of growth in shaken broth culture was confirmed by electron microscope observations (Figs. 4 and 5). After 2 days, empty hyphae were found in both S-77 and S-77F (Figs. 6, 7, 8). These were infrequent in S-77, whereas in S-77F a large part of the mycelium was destroyed and relatively short hyphal segments survived. During the remainder of an 8-day study, the ratio of lysed to unlysed mycelium did not appear to increase on electron-micrographs of S-77. In contrast, the mycelial character of $\mathrm{S}-77 \mathrm{~F}$ was completely destroyed between the second and third days. This was due to further lysis followed 

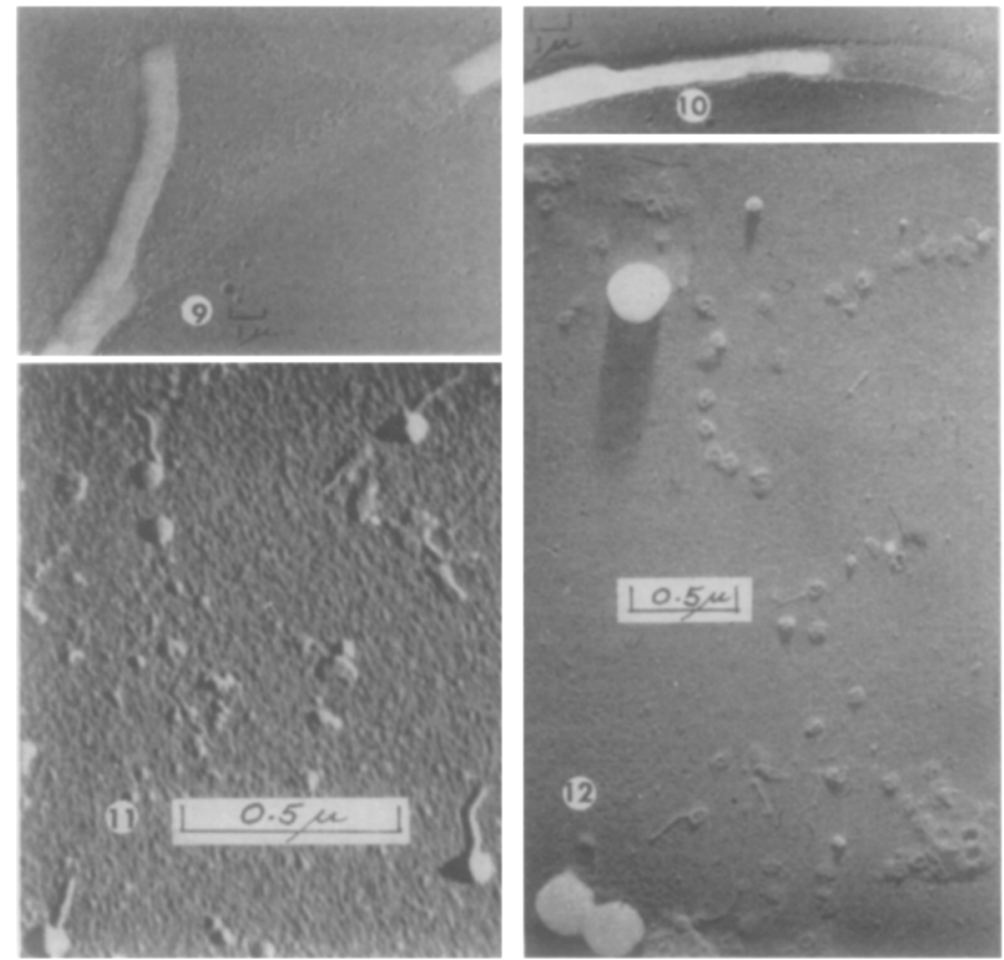

Fig. 9. S-77, 30 hours. Masses of newly formed actinophage within collapsed ghost hyphae and spilled out onto collodion film. Uranium shadowed, $4125 \times$.

Fia. 10. S 77F, 30 hours. Masses of newly formed actinophage within ghost hyphae and spilled out onto collodion film. Uranium shadowed, $4125 \times$.

Fig. 11. Phage from S-77F. Electron micrograph, air-dried on collodion film, uranium shadowed, $45,000 \times$.

FI(a. 12. Portion of droplet pattern of phage preparation from $5-77 \mathrm{~F}$, selected to show abundant ghost phage membranes or "doughnuts." Electron micrograph, sprayed on collodion film with polystyrene latex balls added to spray suspension, $22,500 \times$.

by fragmentation of surviving material. These fragments, branched and unbranched and generally $10 \mu$ to $30 \mu$ in length, persisted and were abundant on electron micrographs made at the end of the study.

The relationship of phage production to collapsed hyphae in both $\mathrm{S}-77$ and S-77F was clearly shown on uranium-shadowed preparations from 30-hour shaken cultures (Figs. 9 and 10). Phage particles appear to have 
been forced out during collapse of the hyphal walls, although many are still retained within the ghost filaments.

Phage of $S-77$ and $S-\gamma 7 F$. An electron micrograph of phage from resuspended pellet of S-77F is shown in Fig. 11. The dense heads are $68 \mathrm{~m} \mu$ to $70 \mathrm{~m} \mu$ in diameter. The long tails were frequently curved or S-shaped, $170 \mathrm{~m} \mu$ long and $20 \mathrm{~m} \mu$ in diameter. Partially flattened heads of less electron opacity, about $80 \mathrm{~m} \mu$ in diameter were seen as well as collapsed or "ghost" forms.

Droplet patterns with latex particles gave an estimate of $2 \times 10^{9}$ virus particles per $\mathrm{ml}$ of lysed and turbid 4-day shaken-culture of S-77F. This material also gave a high count of empty membranes of phage. These were calculated to have been present in the broth at a concentration greater than $1 \times 10^{10}$ particles per ml. Their identity as phage material was established by the presence of a number of representatives complete with tail and with the same size and morphology, except for empty head, as the complete phage particles (Fig. 12). Morphologically these collapsed membranes are similar to the so-called "doughnuts" found in preparations made by rupturing bacterial cells artificially before the time for appearance of the first finished phages, or by inducing premature lysis with proflavine (Levinthal and Fisher, 1952; DeMars et al., 1953). Since, in this case, the material from which the preparation was made lysed "normally" in shaken broth culture, no explanation for the presence of the empty membranes can be given. They may represent either the "ghost" protein membranes from matured phage particles that have lost their nucleic acid contents (Anderson, 1949) or the "doughnut" prephage membranes of Levinthal and Fisher, 1952.

Because high-speed centrifugation $(20,000 \mathrm{~g}$ for one hour) failed to produce a visible pellet of phage with S-77 material, washing and dilution steps used for S-77F phage were omitted. Immediately after removal of the tubes from the centrifuge, all but the last drop of supernatant was quickly withdrawn and $1 \mathrm{ml}$ of distilled water added. Droplets of this concentrate were applied directly to collodion-filmed grids. Such preparations contained an objectionable amount of debris, but served the useful purpose of substantiating other evidence that the "nonlytic" S-77 carries the phage and releases it in small amounts. Examination of several grids and many fields revealed only a small number of tailed particles. Twelve fields, each containing only a single tailed virus particle, were photographed so that the particles could be studied with greater magnification. All tailed particles examined in this way were collapsed, although 
some phage of $\mathbf{S}-77$ found on a few electron micrographs of mycelial material was not collapsed (Fig. 9).

More than 200 separate isolates of Streptomyces from 14 soils were tested for susceptibility to lysis by the phage. All tests were negative. Efforts to free S-77 of its phage have been unsuccessful.

\section{DISCUSSION}

The demonstration of phage in all variants of Streptomyces S-77 provides a probable mechanism for the variability of this soil isolate. The soft, asporogenous colony types and turbid broth cultures of S-77L and $\mathrm{S}-77 \mathrm{~F}$ proved to be the result of interrupted lytic breakdown of the mycelium in which extensive fragile ghost areas separated surviving viable hyphal fragments. Two lines of evidence suggest that the lysed areas were sites of phage release: (a) high phage count in electron micrographs was always associated with large numbers of ghost areas, (b) electron micrographs showed phage particles within and spilled out of ghost segments.

One phenomenon of interest, then, is the presence of phage and occasional ghost hyphae in the normal-appearing parent, S-77. Young mycelium was shown to be susceptible to the phage of S-77L but did not show progressive increase in lysis as a result of its own phage production. S-77 may represent a phage-host system in which all or most of the mycelium is susceptible, but in which premature lysis releases most. of the phage in an immature, noninfective state. This is suggested by the large numbers of "doughnut" particles and tailless particles observed in electron micrographs of $\mathrm{S}-77$ phage preparations. Levinthal and Fisher (1952) have found similar particles by prematurely opening infected Escherichia coli cells a short time ahead of normal phage burst. They found such particles to be noninfective and identified them as the protein "coats" or membranes of phage.

In both S-77 and its more lytic variants we find within a single nonseptate mycelium viable areas of protoplasm resistant to phage action or incapable of maturing phage, coexistent with areas that lyse and release phage into the medium. The immediate question raised by these results is whether or not this system parallels true lysogenic systems now well established for unicellular bacteria. Two of the general criteria for lysogeny appear to be satisfied: (a) host and virus coexist in an equilibrium state through many generations and repeated transfer; (b) release of actinophage by $\mathbf{S - 7 7}$ and its variants through lysis of occasional sections 
of the mycelium appears to parallel the carefully studied situation in lysogenic Bacillus megaterium wherein release of bacteriophage is accompanied by the lysis of certain cells of the culture (Lwoff and Gutmann, 1949a,b,c; Lwoff and Gutmann, 1950; Welshimer, 1951; Clarke and Cowles, 1952; Clarke, 1952). However, from the data now available it cannot be stated with certainty whether S-77 and its variants are lysogenic in the sense that all of the mycelium carries prophage internally.

Lack of a suitable indicator culture, free of phage, has been a most serious handicap in this study of a new soil isolate. Quantitative studies on phage release and infectivity and complete evidence for lysogeny in S-77 are, of course, dependent on the availability of a suitable indicator. Unfortunately such a culture has not been found.

Whatever the mechanism involved, the successful perpetuation of both S-77 and its phage implies an equilibrium between phage production and mycelial growth. There may be shifts in the balance between host and phage without loss of the equilibrium. In the macroscopically normal S-77 the balance favors the host at the expense of limited infective phage production. At the other extreme, soft vegetative colonies or turbid broth cultures result when the balance shifts in the direction of increased effective phage release. In the Nocardia-like S-77L and S-77F, the equilibrium has not been destroyed since both host and phage survive, but the morphology of the host is drastically altered. Significantly, under artificial conditions of culture, the observed shifts for S-77 were mostly in the direction of increased phage release.

\section{ACKNOWLEDGMENTS}

The author wishes to express sincere appreciation to Dr. Kenneth L. Jones for access to his extensive actinomycete collection and for his counsel throughout the course of this work. Thanks are also extended to Dr. Cyrus Levinthal for valuable assistance with the electronmicrography.

\section{REFERENCES}

Anoerson, T. F. (1949). The reactions of bacterial viruses with their host cells. Botan. Rev. 15, 464-505.

BACkUS, R. C., and WILIIAMs, R. C. (1950). The use of spraying methods and of volatile suspending media in the preparation of specimens for electron microscopy. J. Appl. Physiol. 21, 11-15.

Carvajal, F. (1953). Phage problems in the streptomycin fermentation. Mycologia $45,209-234$. 
CLARKE, N. A. (1952). Studies on the host-virus relationship in a lysogenic strain of Bacillus megaterium II. The growth of Bacillus megaterium in synthetie medium. J. Bacteriol. 63, 187-192.

Clarke, N. A., and Cowles, P. B. (1952). Studies on the host-virus relationship in a lysogenic strain of Bacillus megaterium $\mathrm{I}$. The relationship between growth and bacteriophage production in cultures of Bacillus megaterium. I. Bacteriol. $63,177-186$.

IJe Mars, R. I., Luria, S. E., Fisher, H., and Levinthal, C. (1953). The production of incomplete bacteriophage particles by the action of proflavine and the properties of the incomplete particles. Ann. inst. Pasteur 84, 113-128.

Ievinthal, C., and Fisher, R. (1952). The structural development of a bacterial virus. Biochim. et Biophys. Acta 9, 419-429.

LuRia, S. E., Williams, R. C., and BackUs, R. C. (1951). Electron micrographir counts of bacteriophage particles. J. Bacteriol. 61, 179-187.

Lwoff, A., and Gutmane, A. (1949a). Les problèmes de la production du bactériophage par les souches lysogènes. La lyse spontanée du Bacillus megatherium. Compt. rend. 229, 789-791.

Lwoff, A., and Gutmane, A. (1949b). Production discontinue de bactériophages par une souch lysogène de Bacillus megatherium. Compt. rend. 229, 679-682.

Lwoff, A., and Gutmann, A. (1949c). La perpetuation endomicrobienne du bactériophage chez un Bacillus megatherium lysogène. Compt. rend. 230, 154156.

Lwoff, A., and Gutmann, A. (1950). Recherches sur un Bacillus megatherium lysogène. Ann. inst. Pasteur 78, 711-739.

Welshimer, H. J. (1951). Observations on phage output and on anaerobic lysis of lysogenic strains of Bacillus megatherium. J. Bacteriol. 61, 153-159. 